\title{
An In silico Approach to Identify High Affinity Small Molecule Targeting m-TOR Inhibitors for the Clinical Treatment of Breast Cancer
}

\author{
Khushboo Patidar ${ }^{1}$, Umesh Panwar ${ }^{2}$, Sugunakar Vuree ${ }^{3}$, Jajoriya Sweta ${ }^{1}$, \\ Manpreet Kaur Sandhu ${ }^{1}$, Anuraj Nayarisseri1 ${ }^{1,24 *}$, Sanjeev Kumar Singh ${ }^{2 *}$
}

\begin{abstract}
Breast cancer is the most frequent malignancy among women. It is a heterogeneous disease with different subtypes defined by its hormone receptor. A hormone receptor is mainly concerned with the progression of the PI3K/AKT/ mTOR pathway which is often dysregulated in breast cancer. This is a major signaling pathway that controls the activities such as cell growth, cell division, and cell proliferation. The present study aims to suppress mTOR protein by its various inhibitors and to select one with the highest binding affinity to the receptor protein. Out of 40 inhibitors of mTOR against breast cancer, SF1126 was identified to have the best docking score of -8.705, using Schrodinger Suite which was further subjected for high throughput screening to obtain best similar compound using Lipinski's filters. The compound obtained after virtual screening, ID: ZINC85569445 is seen to have the highest affinity with the target protein mTOR. The same result based on the binding free energy analysis using MM-GBSA showed that the compound ZINC85569445 to have the the highest binding free energy. The next study of interaction between the ligand and receptor protein with the pharmacophore mapping showed the best conjugates, and the ZINC85569445 can be further studied for future benefits of treatment of breast cancer.
\end{abstract}

Keywords: Breast cancer- mTOR- Schrodinger Suite- MM-GBSA- Molecular Docking- virtual screening

Asian Pac J Cancer Prev, 20 (4), 1229-1241

\section{Introduction}

\section{Breast cancer}

Breast cancer is the prevalent malignancy among women worldwide (Cidadoet al., 2012). It often described as a heterogeneous disease with different subtypes, defined by its hormone receptor. All the current treatment decision for advanced breast cancer based on these biomarkers (Chan et al., 2005). Despite of the advancement in detection and targeted therapies, the mortality rate remains high because of the development of resistant cell lines. Thus, metastasis breast carcinoma remains an ineradicable disease by modern remedial approaches. So, a better perspective must be needed to thrive new treatment regimen.

According to the American cancer society, around 232,340 recent cases of invasive breast cancer and 39,620 breast cancer deaths are anticipated to happen among US women in 2013 (DeSantis et al., 2014). Over the past two decades, breast cancer occurrence and mortality rates have been increasing rapidly. Breast cancer alone constitutes between $25 \%$ and $15 \%$ of all cancer cases and cancer deaths among women respectively (Torre et al., 2015). Developed countries comprise the number of breast cancer cases and deaths, incidence and mortality rate manifest the availability of the detection and treatment procedure. So, prolonged studies and efforts are required to provide better and easy detection and treatment procedures to all kinds of the population (DeSantis et al., 2014; Torre et al., 2015).

Breast cancer is a complicated and multifactorial disease. Therefore, so many risk factors associated with breast cancer, such as age, body mass index, family history having abnormal BRCA1 or BRCA2 gene, hormonal factors like- menarche at an early age, menopause at a late age, late pregnancy, little or no breastfeeding, oral contraceptive, and hormone replacement therapy (Laamiri et al., 2016). The precise interpretation of predisposing factors might be helpful to the development of the

${ }^{1}$ In silico Research Laboratory, Eminent Biosciences, Madhya Pradesh, ${ }^{4}$ Bioinformatics Research Laboratory, LeGene Biosciences Pvt Ltd., Indore, ${ }^{2}$ Computer Aided Drug Designing and Molecular Modeling Lab, Department of Bioinformatics, Alagappa University, Karaikudi, Tamil Nadu, ${ }^{3}$ Department of Biotechnology, Lovely Faculty of Technology and Sciences, Division of Research and Development, Lovely Professional University, Phagwara, Punjab, India *For Correspondence: anuraj@eminentbio.com,skysanjeev@gmail.com 
novel treatment to prevent breast cancer. The top-most encouraging efforts require understanding the gene, gene-environment, or gene-gene interaction.

The leading cause of cancer is the genetic mutation which occurs as a result of genetic instability and environmental factors. In most of the breast cancer, genetic alteration arises during an individual's lifetime and are present only in specific cells of the breast. These types of changes are called somatic mutation and are not inherited. A different kind of genetic changes, which is classified as germline mutation, are typically inherited from parent to their offspring.

Mutation in the number of genes is involved in causing breast cancer. Regarding high-risk family history, the most important genes are BRCA1 and BRCA2 (Ford et al., 1998). Variation in these two genes can increase the chance of developing the tumors by $80 \%$. Therefore, early recognition of carrier among affected women is pivotal to suggest the specialist to determine the best suitable treatment plan (D’Argenio et al., 2015).

\section{mTOR pathway}

mTOR kinase is an intracellular signaling pathway which controls the various actions of our body such as cell division, cell survival, cellular morphology, protein synthesis and integration of metabolism. mTOR kinase signaling is activated in multiple cancer, guided by variation in the gene-coding receptor tyrosine kinase, Ras, PI3K, and PTEN that is involved in numerous cellular processes (Bhagwat et al., 2011). This protein is a component of two multi-subunit complexes known as mTOR 1 and mTOR2. The mTOR 1 complex is sensitive to anticancer drug Rapamycin, an allosteric inhibitor of mTORC1 results in complete disruption of the complex. The mTOR2 complex is considered as rapamycin-insensitive, regulates actin cytoskeleton by phosphorylating the survival kinase AKT at SER 473. mTOR is mainly activated by some intracellular and extracellular signals such as growth factors, nutrient status, energy metabolism and Oxygen level (O'Reganet al., 2011; Aarthy et al., 2017).

\section{Dysregulation of the mTOR pathway}

The aberrant activation of mTOR pathway identified in most of the cancer including breast cancer and its hyperactivation commonly associated with cell growth, cellular proliferation and neogenesis (García-Echeverría et al., 2010). For instance, in the variety of cancer phosphatase and tensin homologue deleted in chromosome 10 (PTEN) is mutationally inactivated, leads to an increase in mTOR activity. This hyperactivated $\mathrm{mTOR}$ gene results in the production of mRNAs that encode growth factors, cell death inhibitor, angiogenesis factors, cell growth inducer which overall promote carcinogenesis. Therefore, mTOR must be specifically targeted as an anticancer therapy for the treatment of cancer (Dowling et al., 2007). Overall features of the mTOR signaling pathway have provided a higher level of interest in targeting mTOR as a potential therapeutic agent for effective treatment (Xie et al., 2016).

\section{Materials and Methods}

\section{Methodology \\ System Configuration}

The present study was performed using a Schrödinger's Drug Discovery Suite 2015 on the platform of Cent OS Linux 6.5 version. Molecular Dynamics simulations were carried out using an academic version of Desmond 2015.

\section{Selection of Protein Target and known inhibitors}

The three-dimensional structure of the target protein mTOR was retrieved from Protein Data Bank - PDB (www. rcsb.org) with PDB ID: 5H64. Established inhibitors for mammalian target of rapamycin (mTOR) were explored using NCBI's PubChem compound database (Chandrakar, B. et al., 2013; Rao DM. et al., 2010). The total of 40 compounds were selected to consider the best-anchored compound. The selected inhibitors along with their PubChem ID are shown in Table 1.

\section{Preparation of Protein}

Targeted three-dimensional structural coordinates was pre-processed using Protein Preparation Wizard module in Schrödinger Suite (Protein preparation wizard, Schrodinger, 2017) (Sharda et al., 2017; Bandaru et al., 2017) by implying the parameters like assigning bond orders, zero-order bonds to metal atoms, selenomethionine to methionine conversion, filling absent hydrogen's, capping termini, side chains and loops, and removing waters beyond $5 \AA$ distance surrounding the co-crystallized ligand (Bandaru et al., 2017; Shameer et al., 2017; Nasr et al., 2015; Khandekar et al., 2016; Singh et al., 2019). Further, tautomerization and protonation states were predicted in favor of ligand at $\mathrm{pH}$ 7.00. Lastly, the protein hydrogen bonds were optimized to renovation the overlying hydrogens and minimized using OPLS-2005 force field with root mean square deviation (RMSD) value of 0.30 A ${ }^{\circ}$ (Jorgensenet al., 1996; Glide, 2015; Reddyet al., 2014; Patidar et al., 2016; Shaheen et al., 2015; Praseetha et al., 2016; Babitha et al., 2015).

\section{Lead Compounds and Database preparation}

All the 40 compounds retrieved from NCBI's PubChem Database and a large chemical library of drug-like compounds - Zinc databases were prepared using the Ligand preparation module (LigPrep, 2017) (Dunna et al., 2015; Dunna et al., 2015; Bandaru et al., 2015) of molecular modeling package with suitable parameters like optimization, ring conformation, 2D to 3D conversion, determination of protomers, tautomers, and ionization states at $\mathrm{pH} 7.0$, along with partial atomic charges using OPLS_2005 force field (Bandaruet al., 2014; Jorgensen et al., 1996; LigPrep, 2015; Sinha et al., 2015).

\section{Active Site Prediction and Receptor Grid Generation \\ Using SiteMap module in Schrodinger Suite, Ligand-Binding site of the receptor was predicted. Based}


on top ranked site score, five potential active sites were analyzed. Site 2 was taken for docking analysis based on highest site score along with presence of hydrophobic and charged amino acids (Sinha et al., 2014; Reddy et al., 2014; Panwar et al., 2017).Using Receptor Grid Generation module in Schrodinger, the grid generation was performed on prepared protein with the help of site score from SiteMap (Bandaru et al., 2014; Bandaru et al., 2013). The atoms of protein were fixed within the default parameters of the radii of Vander Waal's scaling factor of $1 \AA$ with partial charge cut-off of $0.25 \AA$ using OPLS_2005 force field (Vuree et al., 2013).

\section{Molecular Docking}

Firstly, all the selected 40 compounds were docked into the generated grid of prepared protein using Glide XP (extra precision) module, Schrodinger with default parameters. Glide score is used to rank the various poses of ligand in complex with receptor, where the higher negative values reveal strong binding interaction of protein-ligand. Generally, Glide score is calculated based on the equation:

Glide score $=0.065 * \mathrm{vdW}+0.130 *$ Coul + Lipo + Hbond + Metal + BuryP + RotB + Site (1)

Where, $\mathrm{vdW}=$ vanderWaalsenergy, $\mathrm{Coul}=$ Coulomb energy, Lipo $=$ Hydrophobicinteractions,

H-bond=Hydrogenbonds,

Metal $=$ Metalbindingterms,

Bury $\mathrm{P}=$ Penaltyfor buried polargroups,

RotB $=$ Freezingrotatablebonds, Site $=$ Polar interaction inthebindingsite.

Based on the glide docking score the best-docked complex was selected for further studies of virtual screening (Suryanarayanan et al., 2005; Singh et al., 2016).

\section{High throughput Virtual Screening}

Virtual screening is one of the highly advanced computing processes to identify the novel drug candidate from large chemical libraries against biological target. Herein, the trio of HTVS, SP, and XP from Glide, Schrodinger was utilized to filter the best configuration of ligand with highest docking score in every step of virtual screening trio (Aarthyet al., 2017; Suryanarayanan et al., 2005; Singh et al., 2016). Finally, the best ranking compounds were selected using criteria of docking score for further studies of ADMEprediction.

\section{Pharmacophore studies}

Pharmacophore studies involve different types of interactions between ligand and receptor. This study includes $\mathrm{H}$-bond interaction, electrostatic interaction, hydrophobic interaction, and aromatic interaction done by using Accelrys Discovery Studio 3.5 DS Visualizer (Visualizer, Accelrys Inc, 2012; Basak et al., 2016; González-Díaz et al., 2016; Kelotra et al., 2014; Kelotra, et al., 2014; Majhi et al., 2018; Khandelwal et al., 2018; Sharma et al., 2018; Sinha et al., 2018).

\section{ADME and Toxicityprofile}

As per olden research, many of drugs have been failed during clinical studies due to poor ADME/T; it leads to high cost of loss to pharmaceutical companies. Thus, QikProp, Schrodinger was taken forward for profiling the ADME properties of selected hits from screening. It evaluates the drug likeness and pharmaceutical properties such as molecular weight, aqueous solubility $\left(\mathrm{Q}^{\mathrm{P}} \log ^{\mathrm{S}}\right)$, octanol/water $\left(\log ^{\mathrm{P}}\right)$, brain/blood partition coefficient $\left(\mathrm{Q}^{\mathrm{P}} \log ^{\mathrm{BB}}\right)$, CNS, hydrogen bond donors and acceptors along with Lipinski rule of five and Jorgensen rule of three. Following to these, the Human intestinal absorption (HIA), Blood brain barrier (BBB), AMES toxicity and LD50 were also generated to check compounds toxicity effect using online web server tool admetSAR (QikProp 2015; Cheng et al., 2012).

\section{MM-GBSA (Molecular Mechanics, the Generalized Born model and Solvent Accessibility) - free binding energy calculation}

MM-GBSA, an efficient computational approach in Prime module of Schrodinger suit 2015 (Schrodinger, Inc., LLC, New York, USA), is worthwhile to calculate the relative binding free energy and to improve docking score after docking analysis from bio-molecular system. The binding free energy determines by $\Delta \mathrm{G}_{\text {bind }}$, represented by following equation (Prime, Schrodinger, 2015; Lyne et al., 2006):

$$
\Delta \mathrm{G}_{\text {bind }}=\Delta \mathrm{E}+\Delta \mathrm{G}_{\text {solv }}+\Delta \mathrm{G}_{\mathrm{SA}}
$$

Where, $\Delta \mathrm{G}_{\text {bind }}=$ Binding Free Energy, $\Delta \mathrm{E}=$ Difference of energy minimization between receptorligand complex $\&$ the energies of receptor and ligand Where, $\Delta \mathrm{E}=\mathrm{E}_{\text {complex }}$ $\mathrm{E}_{\text {receptor }}-\mathrm{E}_{\text {ligand }}, \Delta \mathrm{G}_{\text {solv }}=$ Difference of electrostatic solvation energy of the receptor-ligand complex $\&$ the energies of receptor and ligand Where, $\Delta \mathrm{G}_{\text {solv }}=\mathrm{G}_{\text {solv(complex) }}-\mathrm{G}_{\text {solv }}$ (receptor) $-\mathrm{G}_{\text {solv (ligand) }}, \Delta \mathrm{G}_{\mathrm{SA}}=$ Difference of Surface area energies of the receptor-ligand complex and the energies of receptor and ligand:

$$
\text { Where, } \Delta \mathrm{G}_{\mathrm{SA}}=\mathrm{G}_{\mathrm{SA}(\text { complex) }}-\mathrm{G}_{\mathrm{SA} \text { (receptor) }}-\mathrm{G}_{\mathrm{SA} \text { (ligand) }}
$$

The Prime module applies a surface generalized Born model which makes use of a Gaussian surface for enhanced demonstration of a solvent accessible surface area (Suryanarayanan et al., 2005; Singh et al., 2016; Prime, Schrodinger, 2015; Lyne et al., 2006).

\section{BOILED-Egg plot}

A Brain or intestinal EstimateD permeation method (BOILED-Egg) is a predictive model which predicts the bioavailability of the drugs over gastrointestinal absorption and brain penetration. There are five parameters which defines the Cartesian coordinates of both ellipses includes MW, TPSA, MLOGP, GI and BBB. Classification of Egg-plot include the yolk (physiochemical space for BBB permeation), the white (physiochemical space for HIA absorption), and the outside grey region stands for the molecule which predicted low absorption and limited brain penetration properties. The molecule which are placed in grey region are counted as remarked (Padmini et al., 2019; Divya et al., 2019; Palak et al., 2019, Trishang et al., 2019). 
To proceed for further analysis of Egg-plot, compound ID of the top three best drug from each of established docked and virtual screened docked was retrieved. Observable result were analyzed based on the different parameters used for Egg-plot.

\section{Results}

Herein this study, a highly significant virtual screening process was applied to find out an effective leading compound using Schrodinger Software.

Docking and virtual screening results

Molecular docking of top 40 known inhibitors was identified with great binding interaction within the same active site. Later, SF1126, the best one protein-ligand complexes were analyzed as perfect binding confirmation with better docking score, XP Score, glide energy, and Glide emodel, tabulated (Table 2) which were taken forward for virtual screening process against Zinc NCD

Table 1. List of Inhibitors of mTOR Towards Breast Cancer

\begin{tabular}{|c|c|c|c|}
\hline S1. no & Inhibitors name & PubChem ID & Ref \\
\hline 1 & Temsirolimus, CCI-779 & 6918289 & Chan et al., 2005 \\
\hline 2 & Everolimus,RAD001 & 6442177 & Shavetaet al., 2013 \\
\hline 3 & Rapamycin, sirolimus & 5284616 & Jerusalem et al., (2014) \\
\hline 4 & Ridaforolimus, deforolimus, & 11520894 & HareHarvey et al., 2017 \\
\hline 5 & AZD2014 & 25262792 & Guichard et al., (2015) \\
\hline 6 & AZD8055 & 25262965 & Jordan et al., (2014) \\
\hline 7 & INK 128, MLN0128 & 45375953 & Gokmen-Polar et al., (2012) \\
\hline 8 & $\mathrm{CC}-223$ & 58298316 & Bendell et al., (2015) \\
\hline 9 & Palomid 529 (P529), & 11998575 & Xiang et al., (2011) \\
\hline 10 & OSI-027 & 44224160 & Bhagwat et al., (2011) \\
\hline 11 & Torin 1 & 49836027 & Hall et al., (2012) \\
\hline 12 & PP242 & 25243800 & SparksGuertin (2010) \\
\hline 13 & PP30 & 24905154 & Leung et al., (2015) \\
\hline 14 & KU-0063794 & 16736978 & Lee et al., (2013) \\
\hline 15 & XL388 & 59604787 & Liu et al., (2009) \\
\hline 16 & WYE-125132(WYE-132), & 25260757 & Chen et al., (2016); Yu et al., 2010 \\
\hline 17 & WYE-687 & 25229450 & Yu et al., (2009) \\
\hline 18 & WAY-600 & 25229526 & Yu et al., (2009) \\
\hline 19 & WYE-354 & 44219749 & Yu et al., (2009) \\
\hline 20 & Torin 2 & 51358113 & Liu et al., (2013) \\
\hline 22 & GDC-0349 & 59239165 & Pei et al., (2013) \\
\hline 23 & NVP-BEZ235, BEZ235 & 11977753 & Serra et al., (2008) \\
\hline 24 & PF-04691502 & 25033539 & Wander et al., 2013 \\
\hline 25 & NVP-BGT226 & 57336745 & Markman et al., (2012) \\
\hline 26 & Apitolisib,GDC-0980 & 25254071 & Wallin et al., (2011) \\
\hline 27 & PF-05212384, PKI-587 & 44516953 & Fouqué et al., 2016 \\
\hline 28 & SAR245409 (XL765) & 49867926 & Papadopoulos et al. (2014) \\
\hline 29 & GSK2126458 (Omipalisib) & 25167777 & Munster et al., 2015 \\
\hline 30 & PKI-402 & 44187953 & (Mallon et al., (2010)) \\
\hline 31 & PI-103 & 9884685 & Jang et al., 2015 \\
\hline 32 & Metformin & 4091 & Zakikhani et al., 2007 \\
\hline 33 & Antrocin (AKT/mtor) & 53474706 & Rao et al., 2010 \\
\hline 34 & Piperlongumine & 637858 & Shrivastava et al., 2014 \\
\hline 35 & VS-5584 (SB2343) & 46912230 & Kolev et al., 2014 \\
\hline 36 & PKI-179 & 46947264 & Venkatesan et al., 2010 \\
\hline 37 & Resveratrol & & He et al., 2011 \\
\hline 38 & Osthole & 10228 & Hung et al., 2011 \\
\hline 39 & SF1126 & 66577114 & Mahadevan et al., 2012 \\
\hline 40 & MKC-1 & 5327686 & Schneider et al., 2008 \\
\hline
\end{tabular}




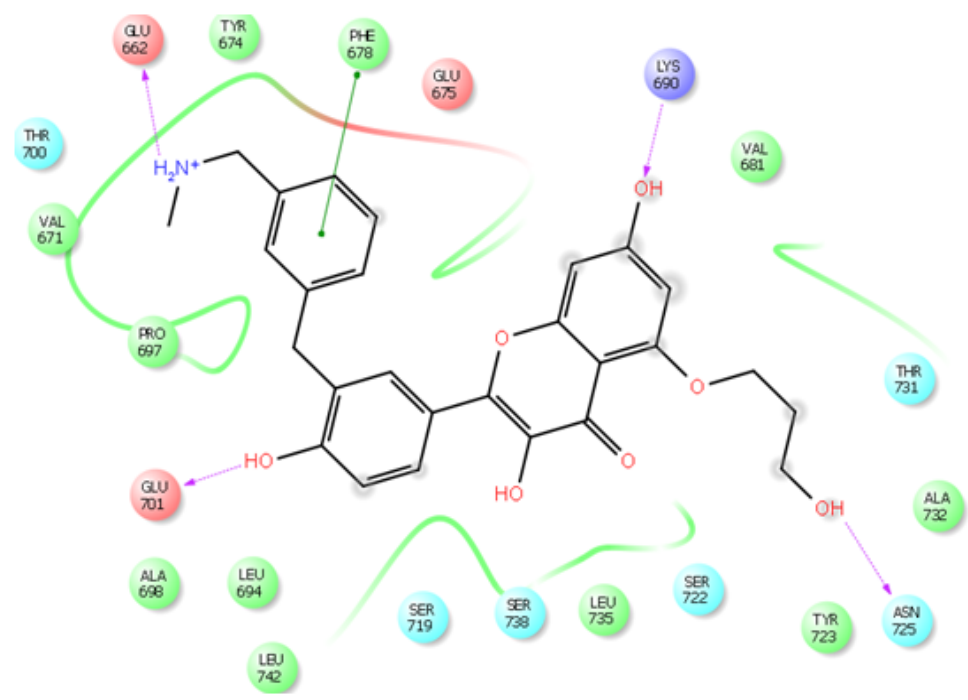

Figure 1. Compound ID:ZINC85569445 Shows High Affinity with mTOR Protein

database. The resulted top 10 compounds with appropriate pharmacological including drug like properties as potent inhibitors against targeted protein with highest docking score, glide score and glide energy, shown (Table 3). The resulted compound ID:ZINC85569445shows the best affinity with the target protein with docking score of $-10.607 \mathrm{kcal} / \mathrm{mol}$. The binding mode analyses of the ZINC85569445 were described in detailed.

Binding mode of Compound ZINC85569445 with the receptor

The ligand ZINC85569445was identified with highest docking score $-10.607 \mathrm{kcal} / \mathrm{mol}$, glide energy -61.060 $\mathrm{kcal} / \mathrm{mol}$, glide Emodel $-82.947 \mathrm{~kJ} / \mathrm{mol}$. Hydrogen bond interactions were identified with the amino acids Glu662, Lys690, Glu701, Asn725; in which, amine group of compound interacted with oxygen of Glu662 with a distance of $2.01 \AA$, three carboxyl group of compound interacted respectively with amino group of Lys690 with a distance of $2.43 \AA$, oxygen of Glu701 with a distance $1.96 \AA$, and oxygen of Asn725 with a distance $2.07 \AA$. Amino acids residues Val671, Tyr674, Phe678, Val681,
Leu694, Pro697, Ala698, Tyr723, Ala732, Leu735, Leu742 were observed as hydrophobic residues. The 2D profile interaction diagram was represented in Figure 1.

\section{Pharmacophore Studies}

Pharmacophore mapping helps to understand the interaction between ligand and receptor molecule. In the active site of target protein, compound ID:ZINC85569445 shows considerable interaction. It shows electrostatic interaction with Ser722 and Lys690, while the H-bond was observed with Glu662, Lys690, Glu701, and Asn725. Figure 4, depicts the aromatic interaction where Phe678, Thr731 actively participated.

\section{Hydrogen Bond interaction between compound ID and} target protein

ZINC85569445 and the target protein mTOR is shown (Figure 2). Green dotted lines represent the Hydrogen interaction between atoms. This interaction involving atoms of the residues Asn725, Glu701, Val67, Ser722, Lys690 of mTOR.

Table 2. Selected Final Compounds from Re-Docking of Knowninhibitors Into the Active Site of the Protein Kinase for Virtual Screening

\begin{tabular}{|c|c|c|c|c|c|}
\hline $\begin{array}{l}\text { Name of } \\
\text { Inhibitors }\end{array}$ & $\begin{array}{l}\text { Docking Score } \\
\text { (kcal mol-1) }\end{array}$ & $\begin{array}{c}\text { Glide } \\
\text { E model } \\
\mathrm{kJ} / \mathrm{mol}\end{array}$ & $\begin{array}{l}\text { Glide Energy } \\
\text { (kcal/ mol) }\end{array}$ & Interactive residues & $\begin{array}{c}\pi-\pi \\
\text { Interaction } \\
\text { Yes/No }\end{array}$ \\
\hline sf1126 & -8.705 & -85.06 & -74.137 & Asp641, Cys679, Ala682, Leu683, Lys690, Asn725 & No \\
\hline WYE-687 & -7.692 & -82.145 & -61.005 & Ser711, Arg718 & No \\
\hline PKI-587 & -7.617 & -88.447 & -45.599 & Leu739 & No \\
\hline NVP-BGT226 & -7.284 & -64.903 & -49.687 & Glu701 & Yes \\
\hline KU-0063794 & -7.174 & -67.057 & -47.904 & Ala666, Ser668 & No \\
\hline GDC-0980 & -7.036 & -82.006 & -59.175 & Ser711, Ser719 & No \\
\hline WYE-354 & -6.97 & -82.607 & -56.887 & $\operatorname{Arg} 718$ & No \\
\hline Torin 2 & -6.839 & -48.904 & -44.086 & Ser738 & No \\
\hline XL388 & -6.826 & -77.967 & -50.654 & - & No \\
\hline GDC-0980 & -6.719 & -78.072 & -57.72 & Ser719 & No \\
\hline
\end{tabular}


Table 3. Structure Based Virtual Screening Results

\begin{tabular}{llccclc}
\hline S. No. & Compound ID & $\begin{array}{c}\text { Docking } \\
\text { Score } \\
(\mathrm{kcal} / \mathrm{mol})\end{array}$ & $\begin{array}{c}\text { Glide } \\
\text { E model } \\
\mathrm{kJ} / \mathrm{mol}\end{array}$ & $\begin{array}{c}\text { Glide Energy } \\
(\mathrm{kcal} / \mathrm{mol})\end{array}$ & $\begin{array}{c}\text { Interactive residues for H-bond between } \\
\text { IN-Ligand }\end{array}$ & $\begin{array}{c}\pi-\pi \\
\text { Interaction } \\
\text { Yes/No }\end{array}$ \\
\hline 1 & ZINC85569445 & -10.607 & -82.947 & -61.06 & Glu662, Lys690, Glu701, Asn725 & Yes \\
2 & ZINC14640443 & -10.437 & -52.385 & -43.829 & Ala682, Leu683, Lys690, Ser719 & No \\
3 & ZINC85489178 & -10.434 & -72.949 & -48.322 & Glu662, Arg716, Ser719, Ser722, Thr731 & No \\
4 & ZINC18208633 & -10.429 & -58.417 & -49.658 & Glu701, Pro715 & No \\
5 & ZINC85569455 & -10.391 & -83.908 & -57.087 & Glu662, Lys690, Glu701 & Yes \\
6 & ZINC85569435 & -10.352 & -76.75 & -58.258 & Glu662, Asn691, Glu701, Thr731 & Yes \\
7 & ZINC06446612 & -10.144 & -102.15 & -63.041 & Glu662, Ser719 & Yes \\
8 & ZINC08694341 & -9.996 & -82.165 & -61.169 & Glu662, Ser719 & No \\
9 & ZINC85569217 & -9.921 & -65.376 & -45.512 & Thr731 & Yes \\
10 & ZINC08791845 & -9.869 & -82.457 & -52.494 & Glu662 & No \\
\hline
\end{tabular}

Electrostatic Interaction between the compounds ID

ZINC85569445 in the active site of mTOR shown in (Figure 3). The red surface of the protein is electrically negative surface; while, the blue surface is electrically positive. The compound is deeply embedded in the cavity of positive and negative amino acids of the target protein mTOR.

Aromatic interactions between the most effective compoundID

ZINC85569445 and mTOR protein shown in (Figure 4). Most favorable region of the aromatic interaction is the blue region (Edge), where Phe678 of mTOR is actively participating in bond formation. While the least favorable region (Face) have less interaction.

\section{Interactions of the compounds ID}

ZINC85569445 in the active site of mTOR shown in [Fig 5]. The residues (Asn725, Glu701, Val67, Ser722, and Lys690) of blue dotted line participating in Hydrogen bond interaction while the residues in green circles forming Van-der Waals. Pink circled residues participating in electrostatic interaction.

\section{ADME profile}

An ADME property of the top 10 hits was calculated by evaluating their physicochemical properties using QikProp, Schrodinger. All the predicted ADME properties are accepted within the standardized range defined for human use such as Molecular weight (MW 130-500), H-Bond donor $(<5.0)$, H-Bond acceptors $(<10.0)$, the octanol/water partition coefficient $\log (-2$ to 6.2$)$, the aqueous solubility $\log (\mathrm{mol} / \mathrm{L})(-6.5$ to 0.5$)$, CNS activity -2 (inactive) to +2 (active Lipinski's rule of five and Jorgensen rule of three), shown in Table 4.

Comparative ADMET profile of the test ligands and the control

Comparative studies of the parameters such as Human intestinal absorption(HIA), Blood-brain barrier (BBB), AMES toxicity, LD50 doses of best established docked compound SF1126 (control) and top three virtual screened ligands (CID: ZINC85569445, CID: ZINC14640443, and CID: ZINC85489178) was done: using R-programming language which is depicted in Figure 6. ADMET parameters such as HIA, BBB, AMES toxicity, and LD50

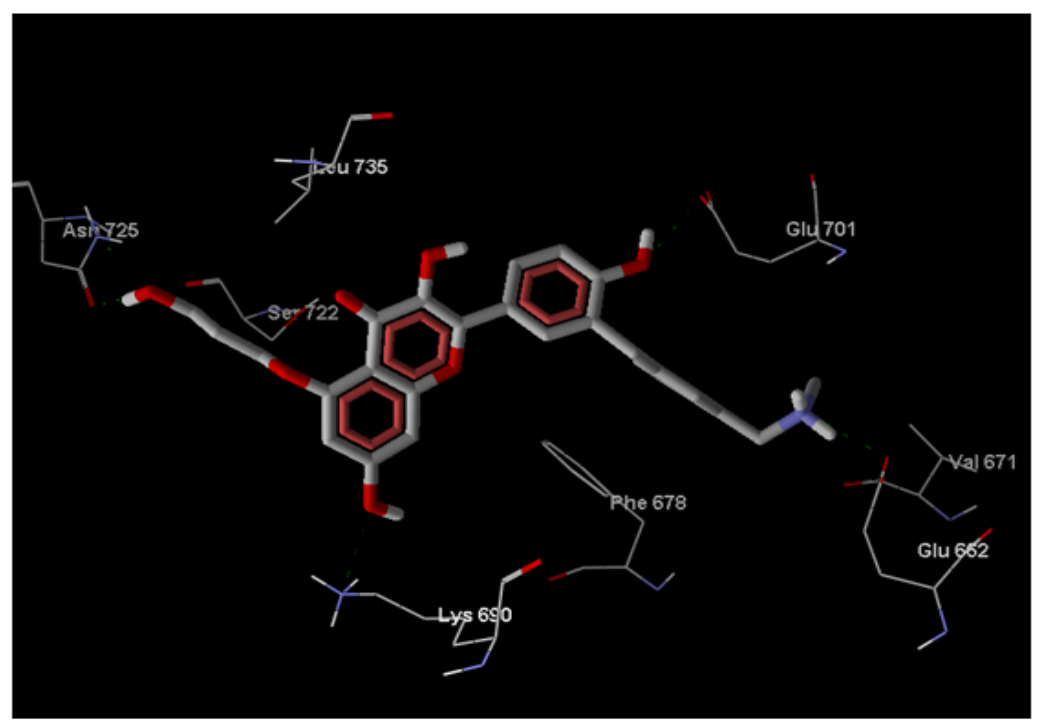

Figure 2. Hydrogen Bond Interaction betweenCompound ID: ZINC85569445 and the Target Protein mTOR

1234 Asian Pacific Journal of Cancer Prevention, Vol 20 


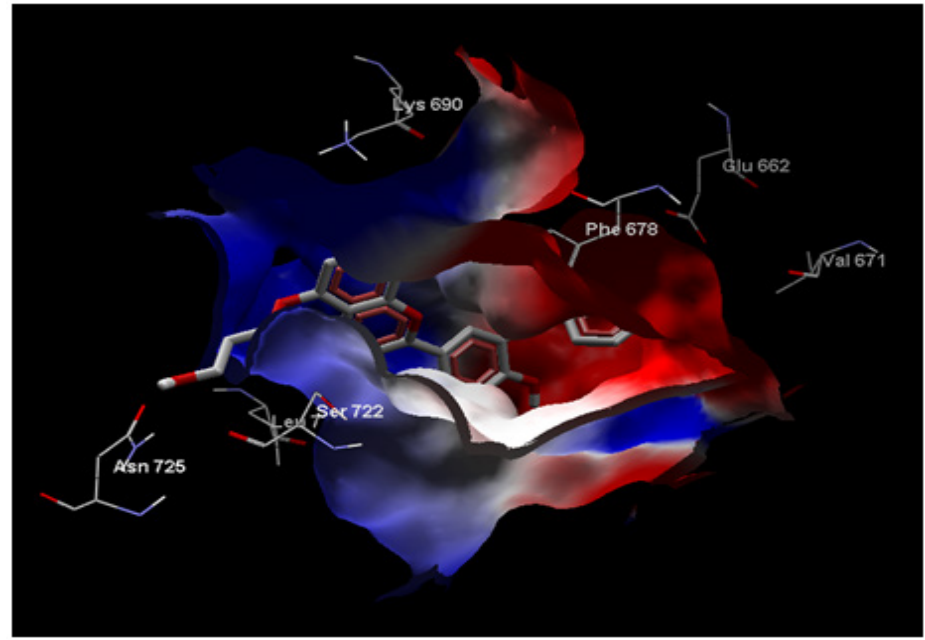

Figure 3. Electrostatic Interaction between the Compounds ID: ZINC85569445 in the Active Site of mTOR

are predicted using online web server tool admetSAR server shown (Table 5). Derived properties revealed that CID: ZINC85569445, CID: ZINC14640443 had the better blood-brain barrier which was higher than the control molecule SF1126. SF1126, CID: ZINC85569445 showed almost equal HIA probability higher than others. Higher HIA denotes the compound could be better absorbed from the intestinal tract after oral administration. Computed result for LD50 dose showed best for SF1126. ZINC85569445 had lower LD50 value compare to another

Table 4. ADME Profile of Screened Hits

\begin{tabular}{lccccccccccc}
\hline S. No. & Compound ID & MW & CNS & HBD & HBA & QPlogPo/w & QPlogS & QPlogBB & QPlogKP & Rule of 5 & Rule of 3 \\
\hline 1 & ZINC85569445 & 477.513 & -2 & 5 & 8.7 & 2.392 & -4.191 & -2.343 & -5.648 & 0 & 2 \\
2 & ZINC14640443 & 288.256 & -2 & 3 & 3.75 & 1.399 & -2.801 & -2.351 & -4.4 & 0 & 0 \\
3 & ZINC85489178 & 470.694 & -2 & 5 & 5.25 & 2.849 & -5.239 & -2.617 & -7.405 & 0 & 2 \\
4 & ZINC18208633 & 388.425 & 0 & 3 & 4 & 3.328 & -3.894 & -0.435 & -4.639 & 0 & 0 \\
5 & ZINC85569455 & 475.54 & -2 & 4 & 6 & 3.703 & -5.389 & -1.943 & -5.472 & 0 & 1 \\
6 & ZINC85569435 & 463.486 & -2 & 5 & 8.7 & 2.099 & -3.808 & -2.116 & -5.593 & 0 & 2 \\
7 & ZINC06446612 & 495.58 & -2 & 1.25 & 7.75 & 4.857 & -5.732 & -1.339 & -0.536 & 0 & 1 \\
8 & ZINC08694341 & 477.562 & -2 & 1.25 & 8.5 & 4.211 & -5.722 & -1.462 & -1.189 & 0 & 1 \\
9 & ZINC85569217 & 476.528 & -2 & 5 & 3.75 & 4.397 & -6.134 & -2.095 & -3.315 & 0 & 2 \\
10 & ZINC08791845 & 439.473 & -1 & 2 & 7 & 4.402 & -6.317 & -0.868 & -1.073 & 0 & 1 \\
\hline
\end{tabular}

MW (Molecular Weight of the molecule), (130.0 to 725.0); CNS, predicted central nervous system activity on a -2 (inactive) to +2 (active) scale; HBD, Hydrogen Bond Donor $=(0.0$ to 6.0); HBA, Hydrogen Bond Acceptor $=(2.0$ to 20.0$) ; \mathrm{QPlogP} \mathrm{o} / \mathrm{w}$ (Predicted octanol/water partition coefficient $)=(-2.0$ to 6.5$)$; QPlogS, (Predicted aqueous solubility, $\log S)=(-6.5$ to 0.5$) ;$ QPlogBB, (Predicted brain/blood partition coefficient $)=(-3.0$ to 1.2); QPlogKP, (Predicted skin permeability, $\operatorname{logKp})=(-8.0$ to -1.0$)$; Rule of 5 , (Number of violations of Lipinski's rule of five $)=($ maximum is 4); Rule of 3, Violations (Number of violations of Jorgensen's rule of three) $=($ maximum is 3 )

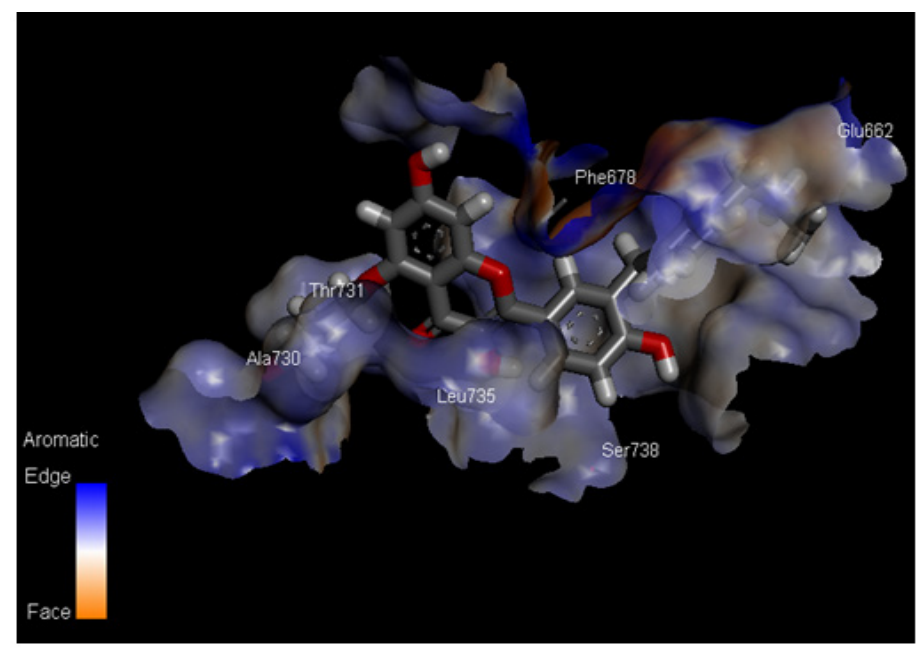

Figure 4. Aromatic Interactions between the most Effective Compound ID: ZINC85569445 and mTOR Protein 
Table 5. Comparative ADMET Profile of Best Established Dock Compound and Test Ligands

\begin{tabular}{lcccccc}
\hline Compound & HIA & BBB & CYP substrate/inhibition & AMES toxicity & Carcinogenicity & LD50 in rat \\
\hline SF1126 & 0.9992 & 0.5096 & Non-substrate/non-inhibitor & 0.5621 & Non-carcinogen & 2.6105 \\
ZINC85569445 & 0.9298 & 0.5101 & Non-substrate/non-inhibitor & 0.7878 & Non-carcinogen & 2.4722 \\
ZINC14640443 & 0.9704 & 0.586 & Non-substrate/inhibitor & 0.663 & Non-carcinogen & 1.9822 \\
ZINC85489178 & 0.8774 & 0.6136 & Non-substrate/non-inhibitor & 0.7778 & Non-carcinogen & 2.3465 \\
\hline
\end{tabular}

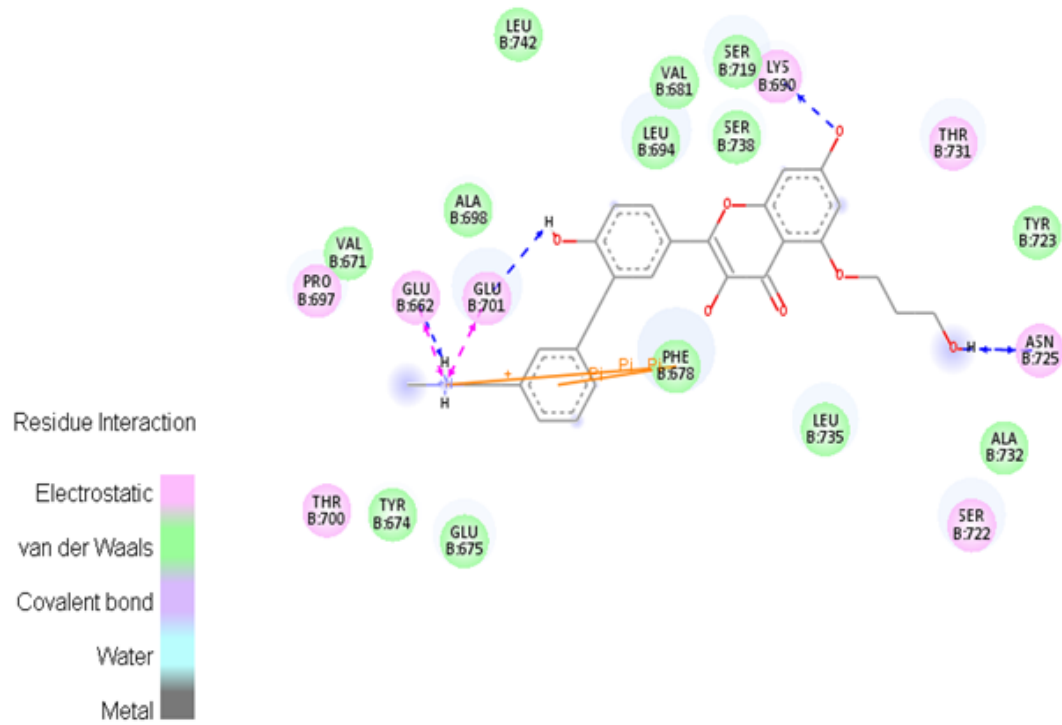

Figure 5. Interactions of the Compound ID: ZINC85569445 in the Active Site of mTOR

compound. CID: ZINC85489178 had almost equivalent LD50 dose to SF1126. Regarding toxicity, the probability of being toxic was lower for SF1126 and ZINC85569445. Ames test employed to test whether a compound is toxic or not. The result of the Ames test revealed that all ligands and control molecule were non toxic.

\section{$M M-G B S A$}

The binding free energy was calculated using a post-scoring method-MMGBSA for the evaluation of molecular docking process. Obtained MMGBSA $(\Delta \mathrm{G}$ bind) ranges from -58.149 to -89.989 , are presented in
Table 4. Results were correlated along with docking score to design a relevant drug like potent inhibitors. Higher binding free energy of the lead defines the greater affinity to bind with the receptor.

\section{BOILED-Egg plot analysis}

To understand the poor pharmacokinetics behaviors and bioavailability of the drug which is crucial for drug discovery process, BOILED-Egg plot is proposed as an accurate model to predict lipophilicity and polarity of the small molecule. Prediction for both BBB and intestinal permeation is translated into a molecular design on

Table 6. Binding Free Energy Analysis Results

\begin{tabular}{|c|c|c|c|c|c|c|}
\hline S. No. & Compound ID/Name & $\Delta \mathrm{G}_{\text {bind }}{ }^{\mathrm{a}}$ & $\Delta \mathrm{G}_{\text {coulomb }}^{\mathrm{b}}$ & $\Delta \mathrm{G}_{\text {covalent }}{ }^{\mathrm{d}}$ & $\Delta \mathrm{G}_{\mathrm{vdW}}{ }^{\mathrm{c}}$ & $\Delta \mathrm{G}_{\mathrm{sol} \mathrm{GB}}$ \\
\hline 1 & SF1126 & -36.926 & -22.993 & 15.799 & -54.637 & 55.852 \\
\hline \multirow[t]{2}{*}{2} & ZINC85569445 & -89.038 & -65.12 & 9.296 & -55.945 & 75.929 \\
\hline & ZINC14640443 & -48.027 & -29.584 & 9.153 & -29.07 & 26.395 \\
\hline 4 & ZINC85489178 & -90.039 & -55.047 & 6.71 & -41.326 & 66.435 \\
\hline 5 & ZINC18208633 & -68.198 & 19.778 & 1.678 & -47.657 & -2.161 \\
\hline 6 & ZINC85569455 & -84.579 & -47.073 & 6.579 & -49.289 & 64.133 \\
\hline 7 & ZINC85569435 & -82.428 & -61.031 & 7.617 & -49.668 & 67.263 \\
\hline 8 & ZINC06446612 & -85.144 & 14.521 & 2.577 & -60.392 & 1.39 \\
\hline 9 & ZINC08694341 & -81.948 & 10.026 & 10.091 & -58.752 & 7.041 \\
\hline 10 & ZINC85569217 & -69.215 & -12.936 & 10.077 & -47.402 & 32.732 \\
\hline 11 & ZINC08791845 & -73.241 & 13.789 & 11.497 & -57.52 & 5.618 \\
\hline
\end{tabular}

Energies in kcal mol-1; a, Free binding energy; b, Coulomb energy contribution to the binding free energy; c, Covalent energy contribution to the binding free energy; d, Van der Waals energy contribution to the binding free energy; e, The generalized born electrostatic solvation energy contribution to the binding free energy. 


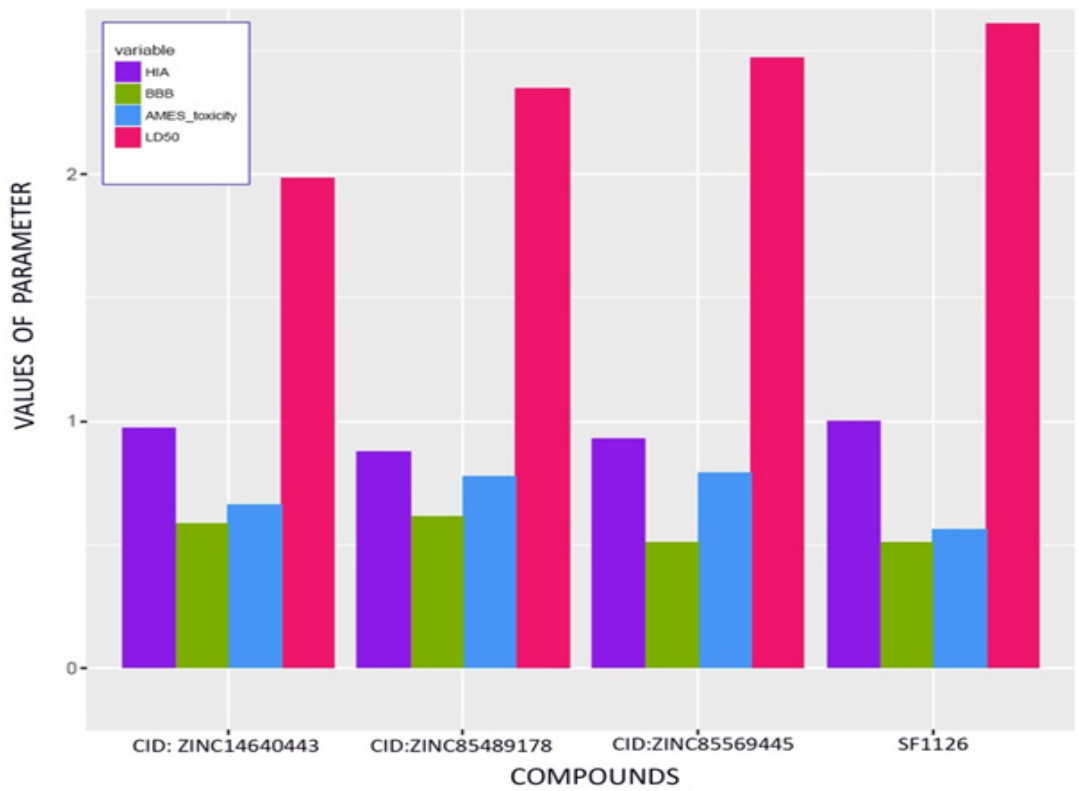

Figure 6. Comparative HIA, BBB, and LD50 of the Established Compound against Virtual Screened Compounds

Table 7. Best Three Compound from -Established Dock Result and Virtual-Screened Dock Result Used for BOILED-Egg Plot

\begin{tabular}{lccccc}
\hline Molecule & MW & TPSA & MLOGP & GI absorption & BBB permeant \\
\hline SF1126 & 852.84 & 344.2 & -6.42 & Low & No \\
WYE-687 & 528.61 & 110.53 & 1.61 & High & No \\
PKI-587 & 615.73 & 128.29 & 1.21 & High & No \\
ZINC85569445 & 478.51 & 136.97 & -2.84 & High & No \\
ZINC14640443 & 288.25 & 118.22 & 0.48 & High & No \\
ZINC85489178 & 471.7 & 104.61 & -3.51 & High & No \\
\hline
\end{tabular}

account of speed, reliability, conceptual clarity and clear graphical output of the model (Table 5) indicates the pharmacokinetics and bioavailability properties of the best six compound; three from established docking and three from virtual screening. It suggests that there is low GI absorption for best pre-established docked compound while high GI absorption for the virtual screening compound. The result itself indicates that there is no BBB

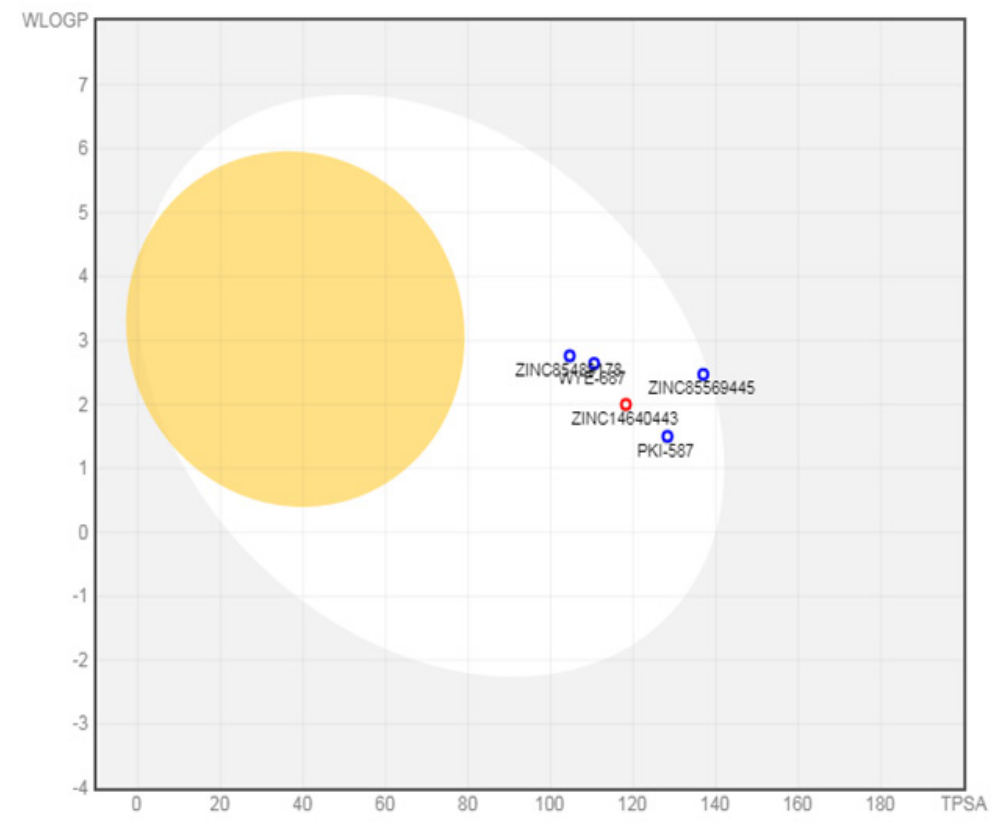

Figure 7. Boiled Egg Plot Predicted for the Best 3 Compounds from Established Drugs and Virtual Screened Drugs 
permeant. All the virtual screened drugs are in white space meaning proper GI-intestinal absorption. BOILED-Egg plot is shown (Figure 7).

\section{Discussion}

Breast Cancer is considered as the prime cause of mortality among women worldwide. Metastatic breast cancer is a diverse disease with different subtypes and irremediable with current treatment regimens (Brouckaert et al., 2017). Based on the data composed by American cancer society, nearly 231,840 recent cases supposed to be diagnosed in 2105 (Ward et al., 2015). One important cause of cancer is the sequential mutation in the number of genes due to the fact of genetic instability and environmental factors (Al-Hajj et al., 2003). mTOR (mammalian target of rapamycin) is a serine/threonine kinase, which controls the various acts of our body such as cell growth, survival, metabolism, is upregulated in various cancer. mTOR is a downstream member of the PI3K/AKT signaling pathway, and its aberrant activation is observed in various human malignant diseases (Vicier et al., 2014). Therefore, mTOR is considered as a desirable target for treatment strategies.

Accordingly, substantial importance is given on PI3K/ AKT/mTOR signaling pathway which has led to the progress of different kinds of inhibitors. In the present study, we found a pre-existing drug against mTOR and technically found SF1126 with the best binding score $-8.705 \mathrm{kcal} / \mathrm{mol}$ against mTOR. Further exploration established from the structure similarity search of SF1126 against ZINC database found various compound with similar properties against mTOR. High-throughput virtual screening performed in this study for structure similarity search which revealed ZINC85569445 with best binding score $-10.607 \mathrm{kcal} / \mathrm{mol}$. In this study the tested compound CID: ZINC14640443 displayed with the lowest binding energy of $-10.607 \mathrm{kcal} / \mathrm{mol}$. The binding energy of the control molecule SF1126 was higher than CID: ZINC14640443 as found in our study, thus CID: ZINC14640443 displayed the much better binding score. ADME profile of both the compounds SF1126(control) and test ligand ZINC85569445 was evaluated using QikProp Schrodinger and all the parameters accepted within the standardized range defined for human use. CID: ZINC14640443 predicted blood-brain barrier 0.51 compare to SF1126 of 0.50 . Carcinogenic profile of both the test ligand and control molecule revealed to be non-carcinogenic.

The effectiveness of the known inhibitors against mTOR has been tested with significant antiproliferative activity while some of them are being under phase II/III clinical trials (Chresta et al., 2010). In spite of having high ability in inhibiting the activity of mTOR, inhibitors are quite inadequate in fighting against cancer. The likelihood, there is the number of reasons such as feedback loop can activate the upstream signaling pathway and promote cell survival and proliferation. The mTOR signaling pathway is essential for healthy cell growth and viability and its inhibition can be destructive to other cells and tissues(Xie et al., 2016). Despite some loopholes, further attention should be given to the understanding of the mTOR signaling pathway and its downstream processing which play a vital role in tumor progression.

In conclusion, this research study focuses on finding a satisfactory lead compound which targets to inhibit the breast cancer. Phosphatidylinositol 3- kinase/AKT/ mammalian target of rapamycin(PI3K/AKT/mTOR) pathway is dysregulated in various cancer along with the breast cancer and is the major signaling pathway which controls the routine activity of our body including cell survival, cell division, etc. In this study, we found many pre-existing compounds against the mTOR protein and technically found SF1126 with the high-affinity properties along with the best binding energy score. Further research progress based on the structural similarity of SF1126 against drug database found that there are several more compound having property against mTOR. Best drug found in this research is ZINC85569445and concluded based on the ADME profile and BOILED-Egg plot prediction.

BOILED -Egg plot is a good informant for lead optimization and following analyses the virtual screened compound ZINC85569445 exhibits interesting pharmacokinetics. Virtual tested drugs are correctly placed in the white region of the egg which indicates that it constitutes preferable compound against mTOR protein. The binding free energy using post scoring method MMGBSA calculated for the evaluation of molecular docking, also concluded the virtual screened compound was having higher affinity with the receptor as compared to pre-exist drug.

Conclusively the lead compound is ZINC85569445 ideal for the study of pharmacophore profile. The compound shows the best affinity among all the pre-exit drugs, and inhibition property against the target mTOR as well as the study of pharmacophore mapping of the compound displayed considerable binding affinity in the active site of protein mTOR. This research encourages suitable opportunity for compound ZINC85569445 to prevent breast cancer.

\section{Consent for publication}

Not applicable.

\section{Ethics approval and consent to participate}

Not applicable.

\section{Competing interests}

The authors declare that they have no competing interests.

\section{Acknowledgements}

SKS thank Department of Biotechnology (DBT), New Delhi (No. BT/PR8138/BID/7/458/2013, dated 23rd May, 2013), DST-PURSE 2nd Phase Programme Order No. SR/PURSE Phase 2/38 (G dated 21.02.2017 and FIST (SR/FST/LSI - 667/2016), MHRD RUSA - Phase 2.0 (grant sanctioned vide letter No. F. 24-51 / 2014 - U, Policy (TNMulti-Gen), Dept. of Edn. Govt. of India, Dt. 09.10.2018) for providing the financial assistance. 


\section{References}

Aarthy M, Panwar U, Selvaraj C, et al (2017). Advantages of structure-based drug design approaches in neurological disorders. Curr Neuropharmacol, 15, 1136-55.

Al-Hajj M, Wicha MS, Benito-Hernandez A, et al (2003). Prospective identification of tumorigenic breast cancer cells. Proc Natl Acad Sci U S A, 100, 3983-8.

Babitha PP, Sahila MM, Bandaru S, et al (2015). Molecular docking and pharmacological investigations of rivastigminefluoxetine and coumarin-tacrine hybrids against Acetyl Choline Esterase. Bioinformation, 11, 378-86.

Bandaru S, Alvala M, Nayarisseri A, et al (2017a). Molecular dynamic simulations reveal suboptimal binding of salbutamol in T164I variant of beta2 adrenergic receptor. PLoS One, 12, e0186666.

Bandaru S, Marri VK, Kasera P, et al (2014). Structure based virtual screening of ligands to identify cysteinyl leukotriene receptor 1 antagonist. Bioinformation, 10, 652-7.

Bandaru S, Ponnala D, Lakkaraju C, et al (2015a). Identification of high affinity non-peptidic small molecule inhibitors of MDM2-p53 interactions through structure-based virtual screening strategies. Asian Pac J Cancer Prev, 16, 3759-65.

Bandaru S, Prasad MH, Jyothy A, et al (2013). Binding modes and pharmacophoric features of muscarinic antagonism and beta2 agonism (MABA) conjugates. Curr Top Med Chem, 13, 1650-5.

Bandaru S, Sumithnath TG, Sharda S, et al (2017b). Helix-Coil transition signatures B-Raf V600E mutation and virtual screening for inhibitors directed against mutant B-Raf. Curr Drug Metab, 18, 527-34.

Bandaru S, Tiwari G, Akka J, et al (2015b). Identification of high affinity bioactive Salbutamol conformer directed against mutated (Thr164Ile) beta 2 adrenergic receptor. Curr Top Med Chem, 15, 50-6.

Basak SC, Nayarisseri A, Gonzalez-Diaz H, et al (2016). Editorial (Thematic Issue: Chemoinformatics Models for Pharmaceutical Design, Part 1). Curr Pharm Des, 22, 5041-2.

Bendell JC, Kelley RK, Shih KC, et al (2015). A phase I dose-escalation study to assess safety, tolerability, pharmacokinetics, and preliminary efficacy of the dual mTORC1/mTORC2 kinase inhibitor CC-223 in patients with advanced solid tumors or multiple myeloma. Cancer, 121, 3481-90.

Bhagwat SV, Gokhale PC, Crew AP, et al (2011). Preclinical characterization of OSI-027, a potent and selective inhibitor of mTORC1 and mTORC2: distinct from rapamycin. $\mathrm{Mol}$ Cancer Ther, 10, 1394-406.

Brouckaert O, Rudolph A, Laenen A, et al (2017). Reproductive profiles and risk of breast cancer subtypes: a multi-center case-only study. Breast Cancer Res, 19, 119.

Chan S, Scheulen ME, Johnston S, et al (2005). Phase II study of temsirolimus (CCI-779), a novel inhibitor of mTOR, in heavily pretreated patients with locally advanced or metastatic breast cancer. J Clin Oncol, 23, 5314-22.

Chandrakar B, Jain A, Roy S, et al (2013). Molecular modeling of Acetyl-CoA carboxylase (ACC) from Jatropha curcas and virtual screening for identification of inhibitors. $J$ Pharm Res, 6, 913-8.

Chang SB, Miron P, Miron A, et al (2007). Rapamycin inhibits proliferation of estrogen-receptor-positive breast cancer cells. J Surg Res, 138, 37-44.

Chen Y, Qian J, He Q, et al (2016). mTOR complex-2 stimulates acetyl-CoA and de novo lipogenesis through ATP citrate lyase in HER2/PIK3CA-hyperactive breast cancer. Oncotarget, 7, 25224-40.
Cheng F, Li W, Zhou Y, et al (2012). admetSAR: a comprehensive source and free tool for assessment of chemical ADMET properties. J Chem Inf Model, 52, 3099-105.

Chresta CM, Davies BR, Hickson I, et al (2010). AZD8055 is a potent, selective, and orally bioavailable ATP-competitive mammalian target of rapamycin kinase inhibitor with in vitro and in vivo antitumor activity. Cancer Res, 70, 288-98.

Cidado J, Park BH (2012). Targeting the PI3K/Akt/mTOR pathway for breast cancer therapy. J Mammary Gland Biol Neoplasia, 17, 205-16.

D'Argenio V, Esposito MV, Telese A, et al (2015). The molecular analysis of BRCA1 and BRCA2: Next-generation sequencing supersedes conventional approaches. Clin Chim Acta, 446, 221-5.

DeSantis C, Ma J, Bryan L, et al (2014). Breast cancer statistics, 2013. CA Cancer J Clin, 64, 52-62.

Divya Jain TU, Shreshtha S, Aishwarya G, et al (2019). Design of novel JAK3 Inhibitors towards Rheumatoid Arthritis using molecular docking analysis. Bioinformation, 15, 68-78.

Dowling RJ, Zakikhani M, Fantus IG, et al (2007). Metformin inhibits mammalian target of rapamycin-dependent translation initiation in breast cancer cells. Cancer Res, 67, 10804-12.

Dunna NR, Bandaru S, Raj Akare U, et al (2015a). Multiclass comparative virtual screening to identify novel Hsp90 inhibitors: a therapeutic breast cancer drug target. Curr Top Med Chem, 15, 57-64.

Dunna NR, Kandula V, Girdhar A, et al (2015b). High affinity pharmacological profiling of dual inhibitors targeting RET and VEGFR2 in inhibition of kinase and angiogeneis events in medullary thyroid carcinoma. Asian Pac J Cancer Prev, 16, 7089-95.

Fouque A, Delalande O, Jean M, et al (2015). A novel covalent mTOR inhibitor, DHM25, shows in vivo antitumor activity against triple-negative breast cancer cells. J Med Chem, 58, 6559-73.

Fouque A, Jean M, Weghe P, et al (2016). Review of PI3K/mTOR inhibitors entering clinical trials to treat triple negative breast cancers. Recent Pat Anticancer Drug Discov, 11, 283-96.

Garcia-Echeverria C (2010). Allosteric and ATP-competitive kinase inhibitors of mTOR for cancer treatment. Bioorg Med Chem Lett, 20, 4308-12.

Gokmen-Polar Y, Liu Y, Toroni RA, et al (2012). Investigational drug MLN0128, a novel TORC1/2 inhibitor, demonstrates potent oral antitumor activity in human breast cancer xenograft models. Breast Cancer Res Treat, 136, 673-82.

Guichard SM, Curwen J, Bihani T, et al (2015). AZD2014, an inhibitor of $\mathrm{mTORC} 1$ and $\mathrm{mTORC} 2$, is highly effective in $\mathrm{ER}+$ breast cancer when administered using intermittent or continuous schedules. Mol Cancer Ther, 14, 2508-18.

Hall BA, Kim TY, Skor MN, et al (2012). Serum and glucocorticoid-regulated kinase 1 (SGK1) activation in breast cancer: requirement for mTORC1 activity associates with ER-alpha expression. Breast Cancer Res Treat, 135, 469-79.

Hare SH, Harvey AJ (2017). mTOR function and therapeutic targeting in breast cancer. Am J Cancer Res, 7, 383-404.

He X, Wang Y, Zhu J, et al (2011). Resveratrol enhances the anti-tumor activity of the mTOR inhibitor rapamycin in multiple breast cancer cell lines mainly by suppressing rapamycin-induced AKT signaling. Cancer Lett, 301, 168-76.

Hung CM, Kuo DH, Chou CH, et al (2011). Osthole suppresses hepatocyte growth factor (HGF)-induced epithelial-mesenchymal transition via repression of the c-Met/Akt/mTOR pathway in human breast cancer cells. J Agric Food Chem, 59, 9683-90. 
Jang NY, Kim DH, Cho BJ, et al (2015). Radiosensitization with combined use of olaparib and PI-103 in triple-negative breast cancer. BMC Cancer, 15, 89.

Jerusalem G, Rorive A, Collignon J (2014). Use of mTOR inhibitors in the treatment of breast cancer: an evaluation of factors that influence patient outcomes. Breast Cancer (Dove Med Press), 6, 43-57.

Jordan NJ, Dutkowski CM, Barrow D, et al (2014). Impact of dual mTORC1/2 mTOR kinase inhibitor AZD8055 on acquired endocrine resistance in breast cancer in vitro. Breast Cancer Res, 16, R12.

Jorgensen WL, Maxwell DS, Tirado-Rives J (1996). Development and testing of the OPLS all-atom force field on conformational energetics and properties of organic liquids. J Am Chem Soc, 118, 11225-36.

Kelotra A, Gokhale SM, Kelotra S, et al (2014a). Alkyloxy carbonyl modified hexapeptides as a high affinity compounds for Wnt5A protein in the treatment of psoriasis. Bioinformation, 10, 743.

Kelotra S, Jain M, Kelotra A, et al (2014b). An in silico appraisal to identify high affinity anti-apoptotic synthetic tetrapeptide inhibitors targeting the mammalian caspase 3 enzyme. Asian Pac J Cancer Prev, 15, 10137-42.

Khandekar N, Singh S, Shukla R, et al (2016). Structural basis for the in vitro known acyl-depsipeptide 2 (ADEP2) inhibition to Clp 2 protease from Mycobacterium tuberculosis. Bioinformation, 12, 92.

Khandelwal R, Chauhan A, Hussain T, et al (2018). Structure-based virtual screening for the identification of high affinity small molecule towards STAT3 for the clinical treatment of Osteosarcoma. Curr Top Med Chem, 18, 2511-26.

Kolev VN, Wright QG, Vidal CM, et al (2015). PI3K/mTOR dual inhibitor VS-5584 preferentially targets cancer stem cells. Cancer Res, 75, 446-55.

Laamiri FZ, Hasswane N, Kerbach A, et al (2016). Risk factors associated with a breast cancer in a population of Moroccan women whose age is less than 40 years: a case control study. Pan Afr Med J, 24, 19.

Lee SL, Chou CC, Chuang HC, et al (2013). Functional role of mTORC2 versus Integrin-Linked Kinase in mediating Ser473-Akt Phosphorylation in PTEN-Negative prostate and breast cancer cell lines. PLoS One, 8, e67149.

Leung EY, Askarian-Amiri M, Finlay GJ, et al (2015). Potentiation of growth inhibitory responses of the mTOR inhibitor everolimus by dual mTORC1/2 inhibitors in cultured breast cancer cell lines. PLoS One, 10, e 0131400.

Liu Q, Thoreen C, Wang J, et al (2009). mTOR mediated anti-cancer drug discovery. Drug Discov Today Ther Strateg, 6, 47-55.

Liu Q, Xu C, Kirubakaran S, et al (2013). Characterization of Torin2, an ATP-competitive inhibitor of mTOR, ATM, and ATR. Cancer Res, 73, 2574-86.

Lyne PD, Lamb ML, Saeh JC (2006). Accurate prediction of the relative potencies of members of a series of kinase inhibitors using molecular docking and MM-GBSA scoring. $J$ Med Chem, 49, 4805-8.

Mahadevan D, Chiorean EG, Harris WB, et al (2012). Phase I pharmacokinetic and pharmacodynamic study of the pan-PI3K/mTORC vascular targeted pro-drug SF1126 in patients with advanced solid tumours and B-cell malignancies. Eur J Cancer, 48, 3319-27.

Majhi M, Ali MA, Limaye A, et al (2018). An in silico investigation of potential EGFR inhibitors for the clinical treatment of colorectal cancer. Curr Top Med Chem, 18, 2355-66.

Mallon R, Hollander I, Feldberg L, et al (2010). Antitumor efficacy profile of PKI-402, a dual phosphatidylinositol 3-kinase/mammalian target of rapamycin inhibitor. Mol Cancer Ther, 9, 976-84.

Markman B, Tabernero J, Krop I, et al (2012). Phase I safety, pharmacokinetic, and pharmacodynamic study of the oral phosphatidylinositol-3-kinase and mTOR inhibitor BGT226 in patients with advanced solid tumors. Ann Oncol, 23, 2399-408.

Munster P, Aggarwal R, Hong D, et al (2016). First-in-human phase I study of GSK2126458, an oral pan-class I phosphatidylinositol-3-Kinase inhibitor, in patients with advanced solid tumor malignancies. Clin Cancer Res, 22, 1932-9.

Nasr AB, Ponnala D, Sagurthi SR, et al (2015). Molecular Docking studies of FKBP12-mTOR inhibitors using binding predictions. Bioinformation, 11, 307.

O'Regan R, Hawk NN (2011). mTOR inhibition in breast cancer: unraveling the complex mechanisms of mTOR signal transduction and its clinical implications in therapy. Exp Opin Ther Targets, 15, 859-72.

Padmini Gokhale APSC, Anushka A, Natasha K, Anuraj N, Sanjeev KS (2019). FLT3 inhibitor design using molecular docking based virtual screening for acute myeloid leukemia. Bioinformation, 15, 104-15.

Palak Shukla RK, Diksha S, Anindya D, Anuraj N, Sanjeev Kumar S (2019). Virtual screening of IL-6 inhibitors for idiopathic arthritis. Bioinformation, 15, 121-30.

Panwar U, Singh SK (2017). Structure-based virtual screening toward the discovery of novel inhibitors for impeding the protein-protein interaction between HIV-1 integrase and human lens epithelium-derived growth factor (LEDGF/p75). J Biomol Struct Dyn, 36, 3199-3217.

Papadopoulos KP, Tabernero J, Markman B, et al (2014). Phase I safety, pharmacokinetic, and pharmacodynamic study of SAR245409 (XL765), a novel, orally administered PI3K/ mTOR inhibitor in patients with advanced solid tumors. Clin Cancer Res, 20, 2445-56.

Patidar K, Deshmukh A, Bandaru S, et al (2016). Virtual screening approaches in identification of bioactive compounds Akin to delphinidin as potential HER2 inhibitors for the treatment of breast cancer. Asian Pac J Cancer Prev, 17, 2291-5.

Pei Z, Blackwood E, Liu L, et al (2013). Discovery and Biological Profiling of Potent and Selective mTOR Inhibitor GDC-0349. ACS Med Chem Lett, 4, 103-7.

Praseetha S, Bandaru S, Nayarisseri A, et al (2016). Pharmacological analysis of vorinostat analogues as potential anti-tumor agents targeting human histone deacetylases: an Epigenetic treatment stratagem for cancers. Asian Pac J Cancer Prev, 17, 1571-6.

Rao DM, Nayarisseri A, Yadav M, et al (2010). Comparative modeling of methylentetrahydrofolate reductase (MTHFR) enzyme and its mutational assessment: in silico approach. Int J Bioinformatics Res, 2, 5-9.

Rao YK, Wu AT, Geethangili M, et al (2011). Identification of antrocin from Antrodia camphorata as a selective and novel class of small molecule inhibitor of Akt/mTOR signaling in metastatic breast cancer MDA-MB-231 cells. Chem Res Toxicol, 24, 238-45.

Reddy KK, Singh P, Singh SK (2014). Blocking the interaction between HIV-1 integrase and human LEDGF/p75: mutational studies, virtual screening and molecular dynamics simulations. Mol Biosyst, 10, 526-36.

Schneider BP, Karwal M, Laufman L, et al (2008). A phase II study of oral MKC-1 for metastatic breast cancer (MBC). J Clin Oncol, 26, 1046.

Selvaraj C, Priya RB, Lee J-K, et al (2015). Mechanistic insights 
of SrtA-LPXTG blockers targeting the transpeptidase mechanism in Streptococcus mutans. RSC Advances, 5, 100498-510.

Serra V, Markman B, Scaltriti M, et al (2008). NVP-BEZ235, a dual PI3K/mTOR inhibitor, prevents PI3K signaling and inhibits the growth of cancer cells with activating PI3K mutations. Cancer Res, 68, 8022-30.

Shaheen U, Akka J, Hinore JS, et al (2015). Computer aided identification of sodium channel blockers in the clinical treatment of epilepsy using molecular docking tools. Bioinformation, 11, 131.

Shameer K, Nayarisseri A, Duran FXR, et al (2017). Improving neuropharmacology using big data, machine learning and computational algorithms. Curr Neuropharmacol, 15, 1058.

Sharda S, Sarmandal P, Cherukommu S, et al (2017). A virtual screening approach for the identification of high affinity small molecules targeting BCR-ABL1 inhibitors for the treatment of chronic myeloid leukemia. Curr Top Med Chem, 17, 2989-96.

Sharma K, Patidar K, Ali MA, et al (2018). Structure-based virtual screening for the identification of high affinity compounds as potent VEGFR2 inhibitors for the treatment of renal cell carcinoma. Curr Top Med Chem, 18, 2174-85.

Shrivastava S, Kulkarni P, Thummuri D, et al (2014). Piperlongumine, an alkaloid causes inhibition of PI3 K/Akt/ mTOR signaling axis to induce caspase-dependent apoptosis in human triple-negative breast cancer cells. Apoptosis, 19, 1148-64.

Singh S, Vijaya Prabhu S, Suryanarayanan V, et al (2016). Molecular docking and structure-based virtual screening studies of potential drug target, CAAX prenyl proteases, of Leishmania donovani. J Biomol Struct Dyn, 34, 2367-86.

Sinha C, Nischal A, Bandaru S, et al (2015). An in silico approach for identification of novel inhibitors as a potential therapeutics targeting HIV-1 viral infectivity factor. Curr Top Med Chem, 15, 65-72.

Sinha C, Nischal A, Pant KK, et al (2014). Molecular docking analysis of RN18 and VEC5 in A3G-Vif inhibition. Bioinformation, 10, 611 .

Sinha K, Majhi M, Thakur G, et al (2018). Computer aided Drug Designing for the identification of high affinity small molecule targeting CD20 for the clinical treatment of Chronic Lymphocytic Leukemia (CLL). Curr Top Med Chem, 18, 2527-42.

Sparks CA, Guertin DA (2010). Targeting mTOR: prospects for mTOR complex 2 inhibitors in cancer therapy. Oncogene, 29, 3733-44.

Suryanarayanan V, Singh SK (2015). Assessment of dual inhibition property of newly discovered inhibitors against PCAF and GCN5 through in silico screening, molecular dynamics simulation and DFT approach. J Recept Signal Transduct Res, 35, 370-80.

Torre LA, Bray F, Siegel RL, et al (2015). Global cancer statistics, 2012. CA Cancer J Clin, 65, 87-108.

Trishang Udhwani SM, Khushboo S, Jajoriya S, et al (2019). Design of PD-L1 inhibitors for lung cancer. Bioinformation, 15, 139-50.

Venkatesan AM, Chen Z, dos Santos O, et al (2010). PKI-179: an orally efficacious dual phosphatidylinositol-3-kinase (PI3K)/mammalian target of rapamycin (mTOR) inhibitor. Bioorg Med Chem Lett, 20, 5869-73.

Vicier C, Dieci MV, Arnedos M, et al (2014). Clinical development of mTOR inhibitors in breast cancer. Breast Cancer Res, 16, 203.

Vinayak S, Carlson RW (2013). mTOR inhibitors in the treatment of breast cancer. Oncology (Williston Park), 27, 38-44, 6, 8 passim.
Vuree S, Dunna NR, Khan IA, et al (2013). Pharmacogenomics of drug resistance in Breast Cancer Resistance Protein (BCRP) and its mutated variants. J Pharm Res, 6, 791-8.

Wallin JJ, Edgar KA, Guan J, et al (2011). GDC-0980 is a novel class I PI3K/mTOR kinase inhibitor with robust activity in cancer models driven by the PI3K pathway. Mol Cancer Ther, 10, 2426-36.

Wander SA, Zhao D, Besser AH, et al (2013). PI3K/mTOR inhibition can impair tumor invasion and metastasis in vivo despite a lack of antiproliferative action in vitro: implications for targeted therapy. Breast Cancer Res Treat, 138, 369-81.

Ward EM, DeSantis CE, Lin CC, et al (2015). Cancer statistics: breast cancer in situ. CA Cancer J Clin, 65, 481-95.

Xiang T, Jia Y, Sherris D, et al (2011). Targeting the Akt/mTOR pathway in Brcal-deficient cancers. Oncogene, 30, 2443-50.

Xie J, Wang X, Proud CG (2016). mTOR inhibitors in cancer therapy. F1000Res, 5.

Yu K, Shi C, Toral-Barza L, et al (2010). Beyond rapalog therapy: preclinical pharmacology and antitumor activity of WYE-125132, an ATP-competitive and specific inhibitor of mTORC1 and mTORC2. Cancer Res, 70, 621-31.

Yu K, Toral-Barza L, Discafani C, et al (2001). mTOR, a novel target in breast cancer: the effect of CCI-779, an mTOR inhibitor, in preclinical models of breast cancer. Endocr Relat Cancer, 8, 249-58.

Yu K, Toral-Barza L, Shi C, et al (2009). Biochemical, cellular, and in vivo activity of novel ATP-competitive and selective inhibitors of the mammalian target of rapamycin. Cancer Res, 69, 6232-40.

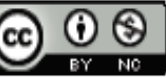

This work is licensed under a Creative Commons AttributionNon Commercial 4.0 International License. 